\title{
Gum Chewing and Cognition: An Overview
}

\author{
Lara Tucha, Janneke Koerts
}

Department of Clinical and Developmental Neuropsychology, University of Groningen, Groningen, The Netherlands. Email: l.i.tucha@rug.nl

Received June 26 ${ }^{\text {th }}$, 2012; revised July 30 ${ }^{\text {th }}$, 2012; accepted August $5^{\text {th }}, 2012$

\begin{abstract}
In recent years, there was a debate about the effects of gum chewing on various aspects of cognitive functioning. In this review, the results of previous studies are presented and summarized. There is a clear indication that gum chewing can improve various aspects of cognitive functioning including memory, attention and both executive and intellectual functioning. However, there is also clear evidence that chewing gum during cognitive tasks can adversely affect task performance. Therefore, it can be concluded that the replication of (beneficial or detrimental) effects of gum chewing on cognition has been proven to be very difficult. Consequently, the robustness of reported effects of gum chewing on cognition has to be questioned. Suggestions for future research are given.
\end{abstract}

Keywords: Gum Chewing; Mastication; Cognition; Memory; Review

\section{Introduction}

In recent years, there was a debate about the effects of gum chewing on cognition. A number of well controlled studies examined the effects of chewing gum on aspects of memory and attention in healthy young adults and found that gum chewing enhances cognitive functioning, in particular with regard to learning and memory. Because of these positive findings, there was quite some enthusiasm among researchers concerning this beneficial impact of gum chewing on cognition. For example, Scholey [1] summarized in a brief review that "Recent reports suggest that enhancement of memory performance while chewing gum is a fairly robust phenomenon" (p. 215). Furthermore, an increase of learning performance of at least $30 \%$ was claimed following gum chewing (Lehrl, as cited in [2]). This enthusiasm might also explain why some researchers interpret non-significant results of a pilot study as an indication of a facilitating effect of gum chewing [3] which had to be rectified following completion of a full-scale study published two years later by the same group [4]. It has been assumed that the cognition enhancing effect of gum chewing is caused by an increased release of insulin and a changed pattern of regional cerebral blood flow, in particular in fronto-temporal regions of the brain [5-7]. These assumptions are consistent with the findings of neuroimaging studies showing that gum chewing resulted in bilateral increases of neuronal activity in the sensorimotor cortex, supplementary motor area, prefrontal cortex, insula, thalamus, and cerebellum [8].

The reports of a performance-enhancing effect of gum chewing attracted the attention of media worldwide. Both national and international media covered these findings in the context of facilitation of children's learning at school and the ban of chewing gum in schools. In fact, if gum chewing really helps memorizing and remembering information as well as increasing the ability to pay attention, one would conclude that gum chewing should not only be allowed during classes but also that chewing gum is strategically used in classes, e.g. in situations in which new and complex material is taught or during exams. An internet search using the terms "school" and "chewing gum" reveals that teachers, principals and school authorities are indeed confronted with this discussion.

However, there is also a considerable number of recent studies that failed to replicate the beneficial effects of gum chewing on cognition. Moreover, an increasing number of studies even demonstrated negative effects of gum chewing on cognition. On this background, it appears reasonable to review the literature to get a clearer understanding of the effects of gum chewing on cognitive task performance.

\section{Method}

To identify relevant literature, searches of Medline and Web of Knowledge were conducted by using the keyword "chewing gum" individually combined with the search terms "cognition", "memory", "learning”, "attention", "spatial" and "executive functions". Furthermore, references from articles and books were searched for additional references. Studies using chewing gum in combination with pharmacological agents (e.g. nicotine) 
were excluded. Furthermore, only the results of standardized cognitive assessments were considered. Results of questionnaires or any other measures of self-evaluation were not included. Studies focusing on the effect of gum chewing on stress were also excluded. Finally, reviews with no original data, and animal studies were excluded. In total, 26 relevant references were identified and included in the present review.

\section{Effects of Gum Chewing on Cognition}

\subsection{Immediate and Delayed Memory}

The terms immediate and delayed recall of information refer to the reproduction of previously learned information at a given period of time. While "immediate recall" usually indicates the reproduction of information immediately after a defined learning period, "delayed recall" refers to the reproduction of information after a prolonged period of time following learning (several minutes to days or even longer).

Word recall. In one of the first studies examining the effects of gum chewing on cognition, Wilkinson and colleagues [5] found that the chewing of a piece of sugar-free chewing gum resulted in a significant improvement of both the immediate and delayed recall of previously learned words (delayed recall after less than an hour; word list used: 15 words, visual presentation, one learning trial). These findings were confirmed by Stephens and Tunney [9] in a study focusing on the role of glucose in the effects of gum chewing. These authors used a word list containing 15 words which were presented auditorily over five trials to participants (delayed recall after less than an hour). Baker and colleagues [10] could only partially replicate these findings (word list used: 15 words, visual presentation, one learning trial). While these authors found no effects on immediate recall, beneficial effects of gum chewing on delayed word recall were observed. It is notably that the assessment of delayed word recall in this study was performed after a delay of 24 hours. This might indicate that retrieval of previously learned information is significantly increased for a prolonged period of time when gum was chewed during learning (and possibly also during recall, please see discussion of context-dependent effects below). Tucha and colleagues [6] performed two experiments to assess the effect of gum chewing on attentional functioning and immediate and delayed word recall (delayed recall after less than an hour; word list used: 15 words, auditory presentation, one learning trial). Data analysis failed to find any facilitating effect of gum chewing on immediate and delayed recall. Miles and Johnson [11] performed two well controlled experiments and also failed to find a facilitative benefit of gum chewing on immediate and delayed word recall (delayed recall after
24 hours; word list used: 15 words, visual presentation, two learning trials). In another experiment the same authors [12] found that chewing gum during learning did not improve subsequent recall (delayed recall after 24 hours; word list used: 15 words, visual presentation, one learning trial), however their data provided some evidence that gum chewing during learning or at recall might improve delayed recall. In another study of the same group [13] even detrimental effects of gum chewing on immediate and delayed recall were observed (delayed recall after 24 hours; word list used: 15 words, visual presentation, one learning trial). In accordance with these findings, Smith [14] also found negative effects of gum chewing on recall (delayed recall after less than an hour; word list used: 15 words, visual presentation, one learning trial). In this study, gum chewing reduced both immediate recall and recognition of the previously learned words. In this context, it is remarkable that a previous study [15] in which the same test was used revealed no indications of any effects of gum chewing. Miles, Charig and Eva [7] applied an immediate recall paradigm in order to assess the effect of gum chewing on long-term memory (word lists used: 15 words, visual presentation, one learning trial). In their paradigm, the authors prevented the participants to maintain recently presented information (words of a word list) within their short-term memory by articulatory suppression (counting backwards after initial learning for a period of 30 seconds). Similar to the previous studies, this experiment failed to find beneficial effects of gum chewing on learning or retrieval of previously learned information. In contrast, Onyper and colleagues [16] reported a positive effect of gum chewing on delayed memory (delayed recall after 3 minutes; task applied under 2 conditions, i.e. with full or divided attention; word lists used: 30 words, visual presentation, one learning trial per condition) but only if participants chewed gum prior to the assessment (experiments 1a and 1b) and not during the assessment (experiment 2). Furthermore, the effect was only observed if the assessments of memory were performed early in the testing session.

Other measures of immediate and delayed recall of information. Wada and colleagues [17] did not find any effect of gum chewing on short-term memory as assessed by a computerized short-term memory task requiring participants to memorize random eight-digit numbers. However, detrimental effects of gum chewing were reported by Kozlov and colleagues [18] who performed a series of well-controlled studies demonstrating that chewing gum impairs immediate recall. By modifying various variables within their experiments, the authors could on the one hand replicate their findings and on the other hand show that the detrimental effect of gum chewing was independent of the material used in the as- 
sessment (letters or numbers; item sequence or item identity) as well as of the modality of presentation (visual or auditory). Moreover, Kozlov and colleagues [18] revealed that the adverse effects of gum chewing on short-term memory are similar to the detrimental effects of finger tapping. With regard to the delayed recall of information, Wilkinson and colleagues [5] found no effects of gum chewing on performance in a delayed picture recognition task (recall after less than an hour; 20 photographic images, visual presentation, one learning trial). Allen and colleagues [4] asked first-year incoming dental students to listen to a videotaped lecture about dental anatomy (50 minutes) and to complete afterwards a multiple choice test about the lecture. Following this assessment, students were asked to read a reading comprehension passage (20 minutes) and to complete a multiple choice test about this text. While the former test represented a measure of specific learning, the latter test was used as a measure of general learning. Despite the fact that the study was well designed and controlled for various variables (e.g. compliance with instructions), the authors failed to find any memorial facilitation of chewing gum. In a study on 4th and 5th grade students of elementary schools, positive effects of gum chewing were found on a story comprehension test [19]. Children were asked to listen to short stories and to memorize as much of these stories as possible. After 5 minutes and 24 hours, children were requested to write down as much as they could remember from these stories. Data analysis revealed that there was no effect of gum chewing on the performance at the 24 hours delayed recall trial. In the 5 minutes delayed recall trial however, children who chewed gum during learning and recall performed significantly better than children without gum at learning, children without gum at recall and children without gum at learning and recall. Smith [20] used short stories in his study and asked participants to recall the stories twice: less than one hour after learning and after a period of two days. There was no effect of gum chewing on recall performance.

Summary of studies examining the effect of gum chewing on memory. In total, 16 studies examined the impact of gum chewing on aspects of immediate and delayed recall. Two studies found beneficial effects of gum chewing on immediate recall of information [5,9] and six studies reported facilitating effects of gum chewing on delayed recall of information $[5,9,10,12,16,19]$. In contrast, one study reported detrimental effects of gum chewing on immediate and delayed recall [13] and two studies on immediate recall only $[14,18]$. Two studies failed to find any effects of gum chewing on both immediate and delayed recall $[6,11]$. In addition, four studies found no evidence of facilitating or detrimental effect of chewing gum on immediate recall $[10,12,15,17]$ and four studies failed to find effects on delayed recall $[4,7,19$, 20].

\subsection{Context-Dependent Effects of Gum Chewing on Memory}

Scholey [21] speculated that Tucha and colleagues [6] failed in their attempt to replicate a facilitating effect of gum chewing on memory because of a shift in context. The term context refers to the impact the environment or internal state of an individual has on information acquisition and retrieval. In general, it was shown that the recall of information is superior when the context during recall resembles the context of learning than when learning and retrieval of information is performed in different contexts $[22,23]$. In their experiments, Tucha and colleagues [6] asked participants to chew the same piece of chewing gum throughout the whole test session. Therefore, the assumption that some properties of the chewing gum might have changed throughout the test period appears quite reasonable (e.g. consistency, texture or flavor of the gum). On the basis of Scholey's [21] assumption, six studies examined whether chewing gum can produce context-dependent effects upon memory functioning. The typical design of these studies was that participants learned word lists (see above) either whilst chewing gum or not chewing gum and that they were requested to recall the words in the same or alternate context. Baker and colleagues [10] were the first to examine context-dependent memory effects of gum chewing. These authors performed two experiments and found that gum chewing can induce context-dependent effects on delayed recall (24 hour delay). A change of context during learning and retrieval appeared to produce adverse effects on recall. Furthermore, these authors found that sucking a piece of chewing gum can also result in context-dependent memory effects. Johnson and Miles [11-13] performed several experiments on this topic but could not find a context-dependent benefit on delayed recall when participants were asked to chew gum during learning and retrieval of information. It appears important to point out that these authors could even not find context-dependent memory effects despite attempts of close replication of the study of Baker and colleagues [10]. In one study, Johnson and Miles [13] even report superior performance of those participants who did not chew gum during both learning and recall. However, in another study Miles and colleagues [7] performed an immediate recall paradigm including articulatory suppression (see above) and observed a context-dependent memory effect of gum chewing. Nevertheless, participants who chewed gum during both learning and recall did not perform better in the memory task than participants who did not chew gum during learning and recall. Overman and colleagues [24] asked participants to either chew a piece of cinnamon 
gum or to suck a sweet with cinnamon flavor during learning and recall. These authors also failed to find any indication of context-dependent memory effects of gum chewing (recall after less than an hour; word list used: two word lists containing 15 words each (concrete versus abstract words), visual presentation, one learning trial).

Summary of studies examining context-dependent effects of gum chewing on memory. Two studies observed that chewing gum can provoke context-dependent effects on long-term memory $[7,10]$. However, four studies failed to replicate context-dependent effects of gum chewing upon memory [11-13,24]. In this context, it appears to be important to emphasize that context-dependent effects of chewing gum on memory did not necessarily mean that memory performance was better when participants chewed gum whilst leaning and recalling information in comparison to their performance when not chewing gum. Reports of context-dependent memory effects indicate that participants in the consistent conditions which are the conditions in which both learning and recall was performed in the same context (both with gum or both without gum) performed better than participants in the inconsistent context conditions (gum during learning and no gum during recall or vice versa).

\subsection{Attention}

Attention is a critical ability that is important for a variety of functions of everyday life including perceptual, motor, emotional and cognitive functioning. Since the quantity of information that can be processed simultaneously is limited, the attention system directs behaviour according to temporal and spatial characteristics or situational requirements [25]. Current models and theories of attention define attention as a multidimensional concept with several distinct components or functions [26-30]. Although different models of attention were proposed, the similarities among them are more striking than the differences between them. Van Zomeren and Brouwer [30] integrated the main characteristics and requirements of various models and devised a multidimensional model of attention which contains the following components: alertness, subdivided into tonic and phasic alertness, vigilance/sustained attention, selective attention, divided attention and strategy/shifting. While tonic alertness refers to a relatively stable level of attention which changes slowly according to diurnal physiological variations of the organism, phasic alertness is the ability to enhance the activation level following a stimulus of high priority. The ability to sustain attention enables a subject to direct attention to one or more sources of information over a relatively long and unbroken period of time. Vigilance, as a special type of sustained attention, is the ability to maintain attention over a prolonged period of time during which infrequent response-demanding events occur. Se- lective attention is defined as the ability to focus attention in the face of distracting or competing stimuli. Divided attention is required to respond simultaneously to multiple tasks or multiple task demands. Shifting refers to the ability to flexibly shift the focus of attention in order to control which information from competing sources will be selectively processed.

Alertness. While one study revealed a negative impact of gum chewing on alertness [6], the remaining two studies examining alertness found no indications of any effects of gum chewing [5,15]. However, an improvement of processing speed was observed in a condition in which gum was chewed prior to testing and in which the actual assessment took place early within the testing session [16].

Sustained attention/Vigilance. A beneficial effect of gum chewing was observed on sustained attention [6]. Chewing gum counteracted the decline of attentional functioning over time which is considered normal in continuous tasks requiring attention [29]. However, Kohler and colleagues [31] reported adverse effects of gum chewing (a piece of Parafilm) on vigilance performance in participants following sleep deprivation. This is confirmed by Tucha and colleagues [32] who also found detrimental effects of gum chewing on vigilance performance in both healthy children (mean age: 10.6 years) and children with attention deficits (mean age: 10.8 years). Taenzer and colleagues [33] showed that 8 to 9 year old children performed more poorly on a sustained attention task than children who did not chew gum. This negative effect was only observed during the first 12 minutes of the test. During the remaining test duration of four minutes children in the no-gum-condition were outperformed by children in the gum-condition indicating a beneficial effect of gum chewing on sustained attention. The importance of time on task performance as a modifying factor of the effects of gum chewing on sustained attention has also been shown on an adult sample [34]. While gum chewing had detrimental effects on sustained attention in earlier stages of a sustained attention task, beneficial effects were observed at later stages of the task. Smith [14] found that his participants performed significantly better on a sustained attention task whilst chewing gum than in the condition without gum despite not taking time-on-task into account. In a previous study, however, Smith [15] could not find such an effect. Furthermore, Wilkinson and colleagues [5] found no effects of gum chewing on vigilance.

Selective attention. Rost and colleagues [2] performed two experiments on large samples of children ( $\mathrm{n}>500$ children each; mean age: 11.0 and 11.4 years respectively) and found in one experiment no effect of gum chewing on selective attention (concentration) but a detrimental effect on selective attention in the other experi- 
ment. Smith [14] observed an advantageous effect of gum chewing. Furthermore, Stephens and Tunney [9] registered an improvement of selective attention induced by gum chewing in one of the three measures these authors applied to assess selective attention. This is confirmed by the results of Sakamoto and colleagues [35] who found improvements of focused attention (as assessed with an auditory oddball paradigm) when participants chewed gum. Three other studies failed to find any effects of gum chewing on selective attention $[5,6,15]$.

Divided attention. Divided attention was examined by Tucha and colleagues [6] who found no effects of gum chewing on this attention function.

Shifting. Shifting of attention was examined in two studies. In one of these studies shifting was significantly impaired by gum chewing [6] while in the other study no effects were found [9].

Summary of studies examining the effect of gum chewing on attention. Twelve studies examined the effects of gum chewing on aspects of attention. The majority of studies focused only on single aspects of attention. A comprehensive assessment of all above mentioned components of attention was so far only performed in one study [6]. Four studies reported that gum chewing provoked improvements of single aspects of attention, i.e. sustained attention/vigilance [6,14] and selective attention $[9,14,35]$. In contrast to these findings, four studies revealed detrimental effects of gum chewing on attention functions such as alertness [6], sustained attention/vigilance [31,32], selective attention [2]) and shifting [6]. Furthermore, five studies failed to find evidence that gum chewing affects alertness [5,15], sustained attention/ vigilance [5,15], selective attention [2,5,6,15], divided attention [6] and shifting [9]. An interesting observation was made by Taenzer and colleagues [33] and Tucha and Simpson [34] who demonstrated that time appeared to be a meaningful factor in the psychodynamics of gum chewing. On the basis of their results, one may assume that chewing gum during task execution might impair performance at early stages but improve performance at late stages of cognitive tasks. In contrast, Onyper and colleagues [16] observed in their study that gum chewing only affected cognition favorably when gum was chewed prior to cognitive assessment and when cognitive assessment was performed within the period of $15-20$ minutes after gum chewing. Our current knowledge, however, is still very limited. In this respect, future research evaluating the effects of gum chewing on cognitive functioning should take time as a mediating factor into consideration.

\subsection{Executive Functions}

Executive functions is an umbrella term encompassing various functions of higher cognition including planning and problem solving, attentional control, cognitive flexibility, working memory and the goal-directed initiation, monitoring and inhibition of actions [36]. In total, seven studies examined the effect of gum chewing on various aspects of executive functioning.

Working memory. Five studies assessed working memory. Working memory is the ability to actively store and manipulate information that is required for complex cognitive tasks. While a few studies $[5,9,16]$ reported beneficial effects of gum chewing on working memory (verbal, spatial and/or numeric working memory), Smith [14] found that gum chewing resulted in an impaired performance on a verbal working memory task. Spatial working memory was not affected by gum chewing in two studies performed by Smith $[14,20]$.

Divergent thinking. Divergent thinking is a fluent, creative and original process of problem solving which aims for the production of as many appropriate solutions to a certain problem as possible. In neuropsychological assessment, divergent thinking is usually assessed with verbal fluency tasks [36] which require the participant to produce as many different words as possible according to a lexical rule (e.g. beginning with a certain letter) or a semantic rule (e.g. belonging to a certain category, such as animals). Studies on verbal fluency functions failed to find any effects of gum chewing on both lexical [9] and semantic verbal fluency [16].

Reasoning. The cognitive process of reasoning allows individuals to go beyond the information given and to generate conclusions from information, assumptions and premises. In two studies, measures of reasoning were applied, however no effects of gum chewing could be demonstrated on both children and adults [2,31].

Cognitive flexibility. Cognitive flexibility refers to an individual's ability to switch cognitive or behavioral responses in order to perceive, process and respond to changing environmental conditions in adaptive ways. Using an anagram task, Torney and colleagues [37] found no effect of gum chewing on cognitive flexibility.

Summary of studies examining the effect of gum chewing on executive functioning. The results of three studies indicated that gum chewing can induce an improvement of working memory $[5,9,16]$. In contrast to these findings, one study found detrimental effects of gum chewing on working memory [14]. Another study by the same author [20] failed to reveal any effect of gum chewing on working memory. No impact of gum chewing was found on divergent thinking $[9,16]$, reasoning $[2,31]$ and cognitive flexibility [37].

\subsection{Spatial Skills}

Spatial skills subsume a number of abilities such as spatial orientation, perception of spatial relations (e.g. between objects), spatial imagination and mental spatial 
manipulation (e.g. mental rotation of a map). Nader and colleagues [38] applied in two experiments a task measuring aspects of spatial perception and spatial imagination but observed no significant effects of gum chewing on these abilities. However, the mean scores measured in both experiments denoted poorer task performance when participants chewed gum.

\subsection{Intellectual Functioning}

The term "intellectual functioning" as it is used here refers to cognitive abilities or scores that cannot be easily classified in the categories of functions as used above. These abilities (mathematical skills) and scores (intelligent quotient) might be highly dependent on and related to some of the above mentioned functions (e.g. working memory).

IQ. Smith [20] demonstrated that gum chewing resulted in improved scores in an intelligence test (Alice Heim 5 Test).

Mathematical skills. While two studies did not find any effects of gum chewing on mathematical skills of children [2,19], Johnston and colleagues [39] could demonstrate that gum chewing during class and homework time (over a period of 14 days) significantly improved math scores and math grades of adolescents (mean age: 14.6 years).

Retrieval of information from general knowledge. Smith [14] applied a semantic processing task which requires participants to decide whether statements were true or false. Data analysis revealed that gum chewing resulted in a reduced speed and accuracy.

Verbal comprehension. Rost and colleagues [2] examined children's understanding of word meanings but found no effects of gum chewing.

Summary of studies examining the effect of gum chewing on intellectual functioning. In conclusion, no effects of gum chewing were observed on verbal comprehension [2]. However, semantic processing was negatively affected by gum chewing [14]. Positive effects were found on an intelligence measure [20]. Since successful completion of this intelligence test depends largely on reasoning skills, this finding may not be in accordance with the findings on reasoning as discussed above. Furthermore, one study reported beneficial influence of gum chewing on math scores [39], while two studies failed to find any effect $[2,19]$.

\section{Discussion}

The present review reveals that there are clear indications that gum chewing can improve various aspects of cognitive functioning including memory, attention and both executive and intellectual functioning. However, it has to be stressed that there is also clear evidence that chewing gum during cognitive tasks can adversely affect task performance. Furthermore, a number of well-controlled studies failed to find any effects of gum chewing on cognition. In this context, it has to be considered that the reported number of studies finding no effects of gum chewing is most likely conservative, since null results are difficult to publish [18]. Therefore, it can be concluded that the replication of (beneficial or detrimental) effects of gum chewing on cognition has been proven to be very difficult. The robustness of reported findings concerning the effects of gum chewing on cognition has to be questioned. This conclusion clearly contradicts a previous overview of the literature in which a facilitation of memory functions by gum chewing has been suggested to be a robust phenomenon [1].

It would be interesting to find out why available studies differ so much in their results, in particular since a number of studies applied very similar designs and test procedures (e.g. word lists). There was a discussion about methodological differences between the earlier studies $[1,21,40,41]$. However, subsequent research which considered variables that were assumed to influence the outcome of studies (e.g. way of presentation of word lists, chewing habits of participants, research design) also failed to replicate the findings of previous studies. In this context, three conclusions appear to be important. First, some authors had difficulties to replicate previous research although their studies were close replications of previous work (e.g. $[10,13])$. Second, the same authors found performance enhancing or decreasing effects of gum chewing on cognition in one of their studies but not in another study (e.g. $[14,20])$. Third, some studies found both positive as well as negative effects of gum chewing on different aspects of cognition within the same sample (e.g. [6,14]). It therefore appears that the variables which were assumed to be responsible for different outcomes of studies are not the crucial ones.

This review demonstrated that a number of studies failed to reach statistical significance indicating positive or negative effects of gum chewing on certain aspects of cognition. Since the classical test theory defines the circumstances that lead to rejection or non-rejection of the null hypothesis and since a non-rejection of the null hypothesis does not express that no differences exist in the population [42,43], one might argue that these studies do not help in the discussion whether gum chewing is beneficial or detrimental with regard to cognitive functioning. However, these studies clearly support the conclusion that the effects of gum chewing are difficult to replicate and not very robust.

Since cognition enhancing effects of gum chewing are possible under certain conditions, the aim of future studies should be to identify these specific conditions, factors and circumstances. In this respect, an interesting research 
question might refer to the role of time as a mediating factor in the psychodynamics of gum chewing. Future research should also try to explain why gum chewing has an impact on some functions but not on others. For example, Wilkinson and colleagues [5] found in the same participants memorial facilitation of gum chewing with regard to verbal memory but not with regard to figural memory. Furthermore, it would be interesting to know whether gum chewing has the potential to facilitate learning of more complex information than short word lists. Word list learning (often with only one learning trial) is a very specific task used in experimental research which does not resemble learning in real life situations. Consequently, current research results lack ecological validity. The study of Johnston and colleagues [39] who examined standardized math scores within the school setting are therefore of particular importance. In case that gum chewing really has this potential, we would know that the effect of gum chewing on cognition is not only interesting from an academic perspective but also from an applied perspective, allowing us to recommend gum chewing as some kind of cognitive enhancer. However, since we lack all this information so far and because detrimental effects of gum chewing were repeatedly documented, gum chewing should not be considered as a learning aid or cognitive enhancer.

\section{REFERENCES}

[1] A. Scholey, "Chewing Gum and Cognitive Performance: A Case of a Functional Food with Function but No Food?” Appetite, Vol. 43, No. 2, 2004, pp. 215-216. doi:10.1016/j.appet.2004.07.004

[2] D. H. Rost, L. Wirthwein, K. Frey and E. Becker, "Steigert Kaugummikauen das Kognitive Leistungsvermoegen? Zwei Experimente der Besonderen Art [Does Chewing Gum Enhance Cognitive Abilities? Two Rather Extraordinary Experiments]," Zeitschrift Fur Padagogische Psychologie, Vol. 24, No. 1, 2010, pp. 39-49. doi:10.1024/1010-0652/a000003

[3] K. L. Allen, D. Galvis and R. V. Katz, "Evaluation of CDs and Chewing Gum in Teaching Dental Anatomy," New York State Dental Journal, Vol. 72, 2006, pp. 30-33.

[4] K. L. Allen, R. G. Norman and R. V. Katz, "The Effect of Chewing Gum on Learning as Measured by Test Performance," Nutrition Bulletin, Vol. 33, 2008, pp. 102-107. doi:10.1111/j.1467-3010.2008.00697.x

[5] L. Wilkinson, A. Scholey and K. Wesnes, “Chewing Gum Selectively Improves Aspects of Memory in Healthy Volunteers,” Appetite, Vol. 38, No. 3, 2002, pp. 235-236. doi:10.1006/appe.2002.0473

[6] O. Tucha, L. Mecklinger, K. Maier, M. Hammerl and K. W. Lange, "Chewing Gum Differentially Affects Aspects of Attention in Healthy Subjects," Appetite, Vol. 42, No. 3, 2004, pp. 327-329. doi:10.1016/j.appet.2004.01.003

[7] C. Miles, R. Charig and H. Eva, “Chewing Gum as Con- text: Effects in Long-Term Memory,” Journal of Behavioral and Neuroscience Research, Vol. 6, 2008, pp. 1-5.

[8] M. Fang, J. C. Li, G. Lu, X. Y. Gong and D. T. Yew, “A fMRI Study of Age-Related Differential Cortical Patterns during Cued Motor Movement,” Brain Topography, Vol. 17, No. 3, 2005, pp. 127-137.

doi:10.1007/s10548-005-4446-5

[9] R. Stephens and R. J. Tunney, "Role of Glucose in Chewing Gum-Related Facilitation of Cognitive Function,” Appetite, Vol. 43, No. 2, 2004, pp. 211-213. doi:10.1016/j.appet.2004.07.006

[10] J. R. Baker, J. B. Bezance, E. Zellaby and J. P. Aggleton, "Chewing Gum Can Produce Context-Dependent Effects upon Memory,” Appetite, Vol. 43, No. 2, 2004, pp. $207-$ 210. doi:10.1016/j.appet.2004.06.004

[11] C. Miles and A. J. Johnson, "Chewing Gum and Context-Dependent Memory Effects: A Re-Examination," Appetite, Vol. 48, No. 2, 2007, pp. 154-158. doi:10.1016/j.appet.2006.07.082

[12] A. J. Johnson and C. Miles, "Chewing Gum and Context-Dependent Memory: The Independent Roles of Chewing Gum and Mint Flavour," British Journal of Psychology, Vol. 99, 2008, pp. 293-306. doi:10.1348/000712607X228474

[13] A. J. Johnson and C. Miles, "Evidence against Memorial Facilitation and Context-Dependent Memory Effects through the Chewing of Gum,” Appetite, Vol. 48, No. 3, 2007, pp. 394-396. doi:10.1016/j.appet.2006.10.003

[14] A. Smith, "Effects of Chewing Gum on Cognitive Function, Mood and Physiology in Stressed and Non-Stressed Volunteers," Nutritional Neuroscience, Vol. 13, No. 1, 2010, pp. 7-16. doi:10.1179/147683010X12611460763526

[15] A. Smith, "Effects of Caffeine in Chewing Gum on Mood and Attention," Human Psychopharmacology-Clinical and Experimental, Vol. 24, No. 3, 2009, pp. 239-247. doi:10.1002/hup.1020

[16] S. V. Onyper, T. L. Carr, J. S. Farrar and B. R. Floyd, "Cognitive Advantages of Chewing Gum. Now You See Them, Now You Don't,” Appetite, Vol. 57, No. 2, 2011, pp. 321-328. doi:10.1016/j.appet.2011.05.313

[17] M. Wada, Y. Hoshi, Y. Iguchi and I. Kida, "Near-Infrared Spectroscopic Study on the Effects of Chewing on Short-Term Memory,” Appetite, Vol. 57, No. 3, 2011, pp. 749-752. doi:10.1016/j.appet.2011.08.018

[18] M. D. Kozlov, R. W. Hughes and D. M. Jones, "GummedUp Memory: Chewing Gum Impairs Short-Term Recall,” The Quarterly Journal of Experimental Psychology, Vol. 65, No. 3, 2012, pp. 501-513. doi:10.1080/17470218.2011.629054

[19] Z. Houcan and W. Li, "Effects of Chewing Gum on Learning and Memory," China Journal of Health Psychology, Vol. 15, 2007, pp. 518-520.

[20] A. Smith, "Effects of Chewing Gum on Mood, Learning, Memory and Performance of an Intelligence Test," Nutritional Neuroscience, Vol. 12, No. 2, 2009, pp. 81-88. doi:10.1179/147683009X423247

[21] A. Scholey, "Further Issues Regarding the Possible 
Modulation of Cognitive Function by the Chewing of Gum: Response to Stephens and Tunney (2004) and Tucha et al. (2004)," Appetite, Vol. 43, No. 2, 2004, pp. 221-223. doi:10.1016/j.appet.2004.08.004

[22] D. R. Godden and A. D. Baddeley, "Context-Dependent Memory in 2 Natural Environments: Land and Underwater," British Journal of Psychology, Vol. 66, 1975, pp. 325-331. doi:10.1111/j.2044-8295.1975.tb01468.x

[23] S. M. Smith and E. Vela, "Environmental Context-Dependent Memory: A Review and Meta-Analysis," Psychonomic Bulletin \& Review, Vol. 8, No. 2, 2001, pp. 203-220. doi:10.3758/BF03196157

[24] A. A. Overman, J. Sun, A. C. Golding and D. Prevost, "Chewing Gum Does Not Induce Context-Dependent Memory When Flavor Is Held Constant," Appetite, Vol. 53, No. 2, 2009, pp. 253-255. doi:10.1016/j.appet.2009.06.013

[25] M. Leclercq, "Theoretical Aspects of the Main Components and Functions of Attention,” In: M. Leclercq and P. Zimmermann, Eds., Applied Neuropsychology of Attention: Theory, Diagnosis and Rehabilitation, Psychology Press, New York, 2002, pp. 3-55.

[26] M. I. Posner and S. J. Boies, “Components of Attention,” Psychological Review, Vol. 78, No. 5, 1971, pp. 391-408. doi:10.1037/h0031333

[27] M. M. Sohlberg and C. A. Mateer, "Introduction to Cognitive Rehabilitation: Theory and Practice," Guilford, New York, 1989.

[28] A. F. Mirsky, B. J. Anthony, C. C. Duncan, M. B. Ahearn and S. G. Kellam, "Analysis of the Elements of Attention: A Neuropsychological Approach,” Neuropsychology Review, Vol. 2, 1991, pp. 109-145. doi:10.1007/BF01109051

[29] R. A. Cohen, "The Neuropsychology of Attention,” Plenum Press, New York, 1993.

[30] A. H. Van Zomeren and W. H. Brouwer, "Clinical Neuropsychology of Attention,” Oxford University Press, New York, 1994.

[31] M. Kohler, A. Pavy and C. Van Den Heuvel, "The Effects of Chewing versus Caffeine on Alertness, Cognitive Performance and Cardiac Autonomic Activity during Sleep Deprivation,” Journal of Sleep Research, Vol. 15, No. 4, 2006, pp. 358-368. doi:10.1111/j.1365-2869.2006.00547.x

[32] L. Tucha, W. Simpson, L. Evans, L. Birrel, T. A. Sontag, K. W. Lange and O. Tucha, "Detrimental Effects of Gum
Chewing on Vigilance in Children with Attention Deficit Hyperactivity Disorder,” Appetite, Vol. 55, No. 3, 2010, pp. 679-684. doi:10.1016/j.appet.2010.10.001

[33] U. Taenzer, A. von Fintel and T. Eikermann, "Chewing Gum and Concentration Performance,” Psychological Reports, Vol. 105, No. 2, 2009, pp. 372-374. doi:10.2466/pr0.105.2.372-374

[34] L. Tucha and W. Simpson, "The Role of Time on Task Performance in Modifying the Effects of Gum Chewing on Attention,” Appetite, Vol. 56, No. 2, 2011, pp. 299301. doi:10.1016/j.appet.2010.12.021

[35] K. Sakamoto, H. Nakata and R. Kakigi, "The Effect of Mastication on Human Processing: A Study Using EventRelated Potentials," Clinical Neurophysiology, Vol. 120, No. 1, 2009, pp. 41-50. doi:10.1016/j.clinph.2008.10.001

[36] M. D. Lezak, D. B. Howieson and D. W. Loring, "Neuropsychological Assessment,” Oxford University Press, New York, 2004.

[37] L. K. Torney, A. J. Johnson and C. Miles, "Chewing Gum and Impasse-Induced Self-Reported Stress,” Appetite, Vol. 53, No. 3, 2009, pp. 414-417. doi:10.1016/j.appet.2009.07.009

[38] I. W. Nader, G. Gittler, K. Waldherr and J. Pietschnig, "Chew on This: No Support for Facilitating Effects of Gum on Spatial Task Performance,” Archives of Oral Biology, Vol. 55, No. 9, 2010, pp. 712-717. doi:10.1016/j.archoralbio.2010.06.008

[39] C. A. Johnston, C. Tyler, S. A. Stansberry, J. P. Moreno and J. P. Foreyt, "Gum Chewing Affects Standardized Math Scores in Adolescents,” Journal of Adolescents, Vol. 35, No. 2, 2012, pp. 455-459. doi:10.1016/j.adolescence.2011.04.003

[40] R. Stephens and R. J. Tunney, "How Does Chewing Gum Affect Cognitive Function? Reply to Scholey (2004)," Appetite, Vol. 43, No. 2, 2004, pp. 217-218. doi:10.1016/j.appet.2004.07.005

[41] O. Tucha, L. Mecklinger, M. Hammerl and K. W. Lange, "Effects of Gum Chewing on Memory and Attention: Reply to Scholey (2004)," Appetite, Vol. 43, No. 2, 2004, pp. 219-220. doi:10.1016/j.appet.2004.08.003

[42] J. Cohen, "Statistical Power Analysis for the Behavioral Sciences,” Erlbaum, Hillsdale, 1988.

[43] E. J. Pedhazur and L. P. Schmelkin, "Measurement, Design, and Analysis: An Integrated Approach,” Lawrence Erlbaum, Hillsdale, 1991. 